\title{
New Hot-Carrier Degradation Mechanism for MOSFET Devices Using Two-Type Interface-State Model
}

\author{
M. Yamaji and T. Itakura
}

Fujitsu Laboratories Limited, 10-1 Morinosato-Wakamiya, Atsugi 243-0197, Japan Tel.: +81 46250 8235, Fax: +81 46250 8804, E-mail: yamaji@han.flab.fujitsu.co.jp

\begin{abstract}
We have studied the characteristics of MOSFET degradation induced by hotcarriers. When the characteristics of drain current degradation $\left(\Delta I_{d}\right)$ are applied to the stress time $(t)$ dependence $\Delta I_{d} \propto t^{n}$, the exponent $n$ is clearly different under different bias conditions. We present a two-type interface-state model composed of deep-energy interface states and shallow-energy interface states which have a different $n$ exponent in order to explain the characteristics of drain current degradation.
\end{abstract}

\section{Introduction}

One of the most important reliability issues in deep-submicron MOSFET devices is the hot-carrier-induced oxide damage which results in drain current degradation. There are several drain current degradation models $[1,2]$ which are used in MOSFET hot-carrier reliability simulation. Most of them are expressed as:

$$
\Delta I_{d}(t) \propto \Delta N_{i t}(t) \propto t^{n}
$$

where $\Delta I_{d}$ is the drain current degradation, $N_{i t}$ is the interface-state density, and $\boldsymbol{t}$ is the stress time. Although this equation assumes a constant exponent $n$ under the same stress condition, as shown in Fig. 1 this exponent is clearly different under different bias conditions. The exponent $\boldsymbol{n}$ mainly depends on the gate voltage, as shown in Fig. 2.

\section{Two-Type Interface-State Model}

In order to explain this gate voltage dependence of exponent $n$, we present a new interface-state model called the two-type interface-state model. In this model, two types of interface states are assumed. One is the deep interface state, which exists in the bandgap and correspond to the conventional model; the other is the shallow interface state, which exists in the conduction band. These two-types of interface states have different $\boldsymbol{n}$ exponents in Eq. 1. The exponent of the deep interface state

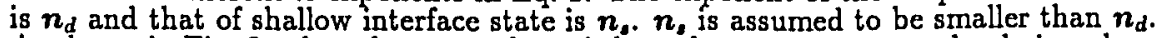
As shown in Fig. 3, when the gate voltage is low, electrons are trapped only in a deep interface state. As the gate voltage is increased, which means that the Fermi level is higher, some electrons begin to be trapped in a shallow interface state. As a result, 
the total exponent $\boldsymbol{n}$ of the interface states is between $\boldsymbol{n}_{\boldsymbol{d}}$ and $\boldsymbol{n}_{\boldsymbol{d}}$ and is smaller at higher gate voltages, as shown in Fig. 2.

Figure 4 shows the interface-state spatial density distribution obtained from chargepumping measurement. Only the deep interface states are observed by charge-pumping measurement[3]. Therefore, the calculated drain current degradation, which is obtained by using the interface states in Fig. 4 , is smaller than the experimental one, particularly in the high gate voltage region. Then the shallow interface-state energy distribution is decided in order to fit the characteristics of drain current degradation of the experiment in the high gate voltage region. Figure 5 shows the interface state energy distribution which includes the shallow interface states. The distribution has also been obtained from several other experiments $[4,5]$. In the two-type interface-state model, the interface-state density distribution $N_{t}(x, \varepsilon, t)$ is expressed as:

$$
\begin{aligned}
& N_{t}(x, \varepsilon, t)=f(x) D(\varepsilon, t)
\end{aligned}
$$

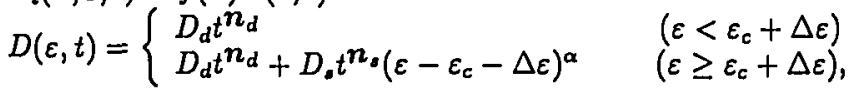

where $f(x)$ is the stress-induced interface-state spatial density distribution obtained from charge-pumping measurement, $D(\varepsilon, t)$ is the density of interface states, $\varepsilon_{c}$ is the bottom of the conduction band, $D_{d}$ and $\boldsymbol{n}_{d}$ are the deep interface-state's parameters, $D_{n}, \Delta \varepsilon, n_{a}$ and $\alpha$ are the shallow interface-state's parameters, and $x, \varepsilon$, and $t$ are the lateral location from the gate center, energy, and stress time, respectively. The drain current degradation is calculated by the device simulator by considering the interface-state distribution of Eq. 2.

\section{Results and Discussion}

As shown in Fig. 6, the drain current degradation using the conventional interfacestate model is lower than the measurement in the high drain region, but the degradation using the two-type interface-state model agrees well with the measurement. The two-type interface-state model satisfactorily represents the stress time and the gate voltage dependence of the drain current degradation (Figs. 7,8 ).

\section{Conclusion}

We presented the two-type interface-state model using Eq. 2 from the characteristics of MOSFE'T degradation induced by hot-carriers. The model satisfactorily represents the stress time dependence of the drain current degradation under several bias conditions.

\section{Acknowledgement}

The authors would like to thank Mr. Ueda and Mr. Ikeda for providing us with nMOSFETs for the hot-carrier stressed experiment of this work.

\section{References}

[1] C. Hu, S. C. Tam, F. Hsu, P. Ko, T. Chan, and K. W. Terrill, "Hot-ElectronInduced MOSFET Degradation - Model, Monitor, and Improvement", IEEE Trans. Electron Devices, ED-32, p.375, 1985.

[2] K. N. Quader, C. C. Li, R. Tu, E. Rosenbaum, P. K. Ko, and C. Hu, "A Bidirectional NMOSFET Current Reduction Model for Simulation of Hot-CarrierInduced Circuit Degradation", IEEE Trans. Electron Devices, ED-40, p.2245, 1993. 
[3] G. Groeseneken, H. E. Maes, N. Beltran, and R. F. De Keersmaecker, "A Reliable Approach to Charge-Pumping Measurements in MOS Transistors" IEEE Trans. Electron Devices, ED-31, p.42, 1984.

[4] H. Sakai, K. Hoh, and T. Sugano "Determination of Interface-State Density and Mobility Ratio in Silicon Surface Inversion Layers", IEEE Trans. Electron Devices, ED-17, p.892, 1970.

[5] A. Schwerin, W. Hänsch, and W. Weber, "The Relationship Between Oxide Charge and Device Degradation: A Comparative Study of $n$ - and p-Channel MOSFET's" IEEE Trans. Electron Devices, ED-34, p.2493, 1987.

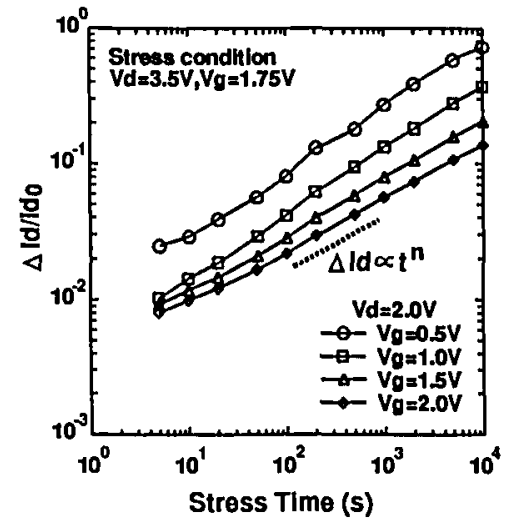

Fig. 1: Stress time dependence of the drain current reverse-degradation. $I_{d}$ was measured at $V_{d}=2 \mathrm{~V}$.

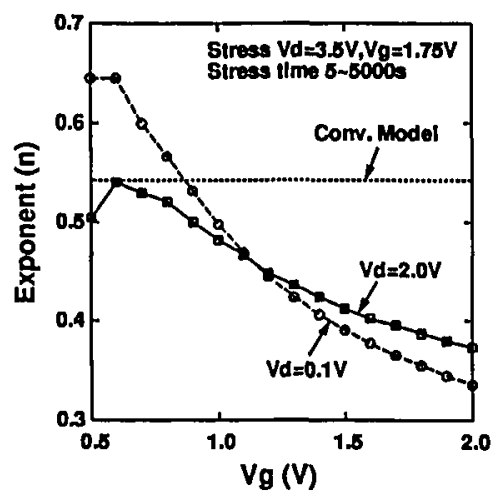

Fig. 2: Gate voltage dependence of reverse-degradation exponent $\boldsymbol{n}$. The dashed and solid lines are from measurements at $V_{d}=0.1 \mathrm{~V}$ and $V_{d}=2.0 \mathrm{~V}$, and the dotted line is from the conventional interface-state model.

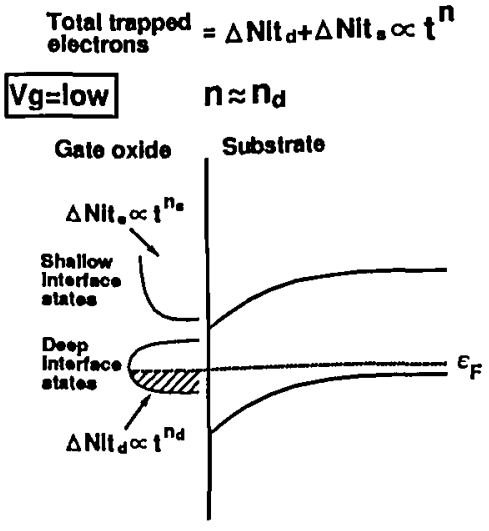

\section{Vg=high $n_{s}<n<n_{d}$}

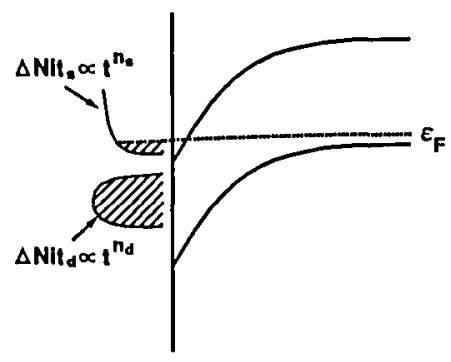

Vg=very high $n \approx n_{s}$

Fig. 3: Schematic diagram of the two-type interface-state model and gate voltage dependence of degradation exponent $n$. 


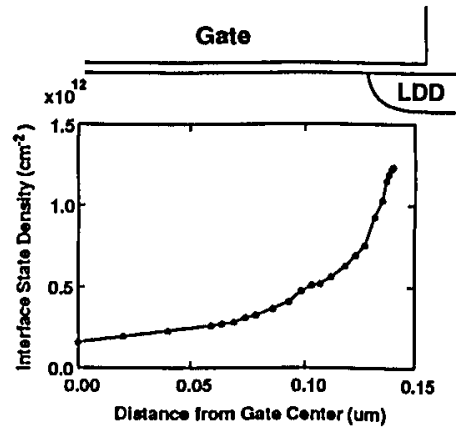

Fig. 4: Interface-state spatial density distribution generated by hot carriers. This distribution was obtained from chargepumping measurement.

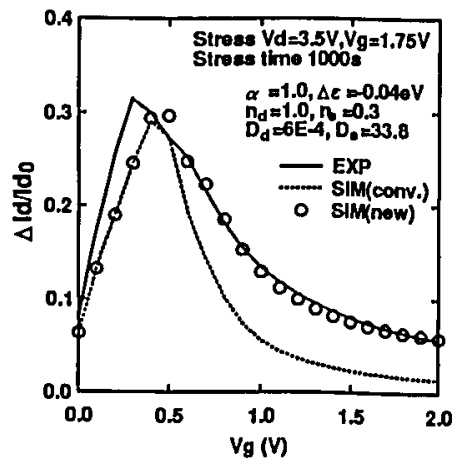

Fig. 6: Drain current reverse-degradation rate versus gate voltage. Open circles are from measurements, the dotted line is from the conventional model, and the solid line is from the two-type interface-state model.

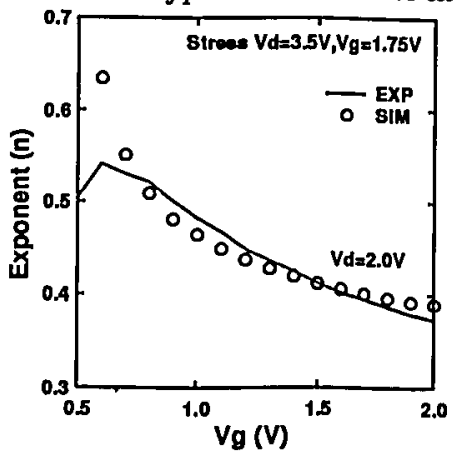

Fig. 8: Gate voltage dependence of reversedegradation exponent $\boldsymbol{n}$. The solid line is from measurements and the open circles are from the two-type interface-state model.

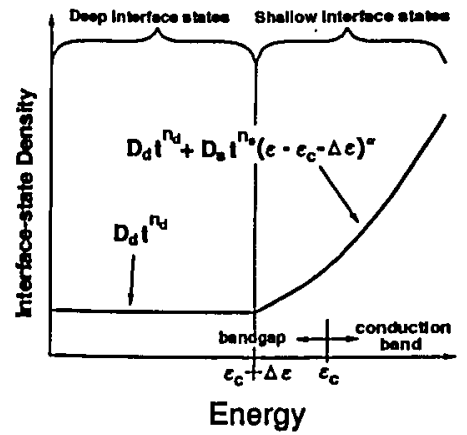

Fig. 5: Interface-state energy distribution (Eq. 2) of two-type interfacestate model.

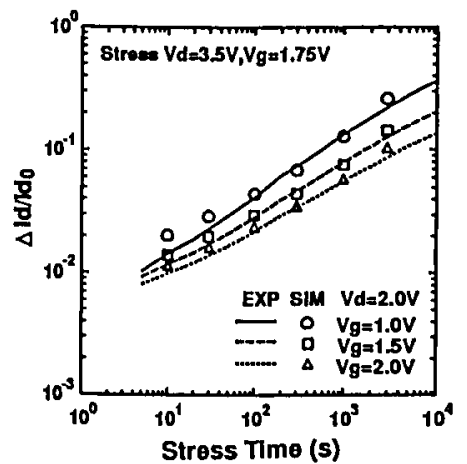

Fig. 7: Stress time dependence of drain current degradation. $I_{d}$ was measured at $V_{d}=2 \mathrm{~V}$. 